\title{
DOKUMENTATIONEN
}

\section{Evaluation von BFD, FSJ und FÖJ: \\ Erste Ergebnisse aus den Befragungen der Freiwilligen}

\author{
Dr. Jens Kreuter
}

Bis Mai 2014 Leiter des Arbeitsstabes Freiwilligendienste im Bundesministerium für Familie, Senioren, Frauen und Jugend (BMFSFJ), Bonn | jens.kreuter@bmfsfj.bund.de

Zur zielgerichteten Weiterentwicklung der Jugendfreiwilligendienste FSJ und FÖJ und des BFD wird im Auftrag des Bundesministeriums für Familie, Senioren, Frauen und Jugend (BMFSFJ) seit Herbst 2012 eine umfassende Evaluation durchgeführt. ${ }^{1}$ Die seit Jahrzehnten bewährten Jugendfreiwilligendienste, das Freiwillige Soziale Jahr (FSJ) und das Freiwillige Ökologische Jahr (FÖJ) sind Angebote für junge Menschen bis zum 27. Lebensjahr. Zur nachhaltigen Stärkung der Jugendfreiwilligendienste werden aktuell rund 50.000 Plätze im FSJ/FÖJ durch den Bund gefördert. Auch der im Juli 2011 eingeführte Bundesfreiwilligendienst (BFD) ist mit bis zu 49.000 Freiwilligen im Dienst (BAFzA 2013) ein enormer Erfolg. Er richtet sich an Frauen und Männer aller Altersgruppen und eröffnet erstmals auch der Zielgruppe der über 27-jährigen Zugang zu einem geregelten Einsatz in einem Freiwilligendienst. BFD und FSJ/FÖJ haben als gesetzlich geregelte Freiwilligendienste und besondere Form des bürgerschaftlichen Engagements positive Wirkungen für den gesellschaftlichen Zusammenhalt und in den jeweiligen vor allem sozialen und ökologischen Einsatzbereichen. Sie leisten als pädagogisch begleitete Bildungs- und Orientierungsangebote einen wichtigen Beitrag zur Persönlichkeitsentwicklung der Freiwilligen und zum personalen, sozialen und fachlichen Kompetenzerwerb. Freiwilligendienste sind damit auch eine Zeit der persönlichen und beruflichen Orientierung für die Freiwilligen und des Einsatzes für die Gesellschaft.

Gegenstand der Untersuchungen sind natürlich ganz besonders die Freiwilligen selbst: Wer sind sie, mit welcher Motivation treten sie ein solches Engagement an, was erhoffen sie sich davon, und werden diese Hoffnungen erfüllt? Das sind nur einige Fragen, mit denen sich die Evaluation befasst. Es geht aber auch um eine 
Analyse der Zielgruppen, Rahmenbedingungen und Strukturen der Freiwilligendienste BFD, FSJ und FÖJ. Die drei zu evaluierenden Formate weisen inhaltliche, strukturelle und hinsichtlich der Zielgruppe große Übereinstimmungen auf. Insbesondere durch die Altersöffnung im BFD waren hier jedoch neue Entwicklungen zu erwarten, so dass dieser neuen Gruppe von Freiwilligen, den sogenannten „Ü 27“, (laut monatlicher Statistik des Bundesamtes für Familie und zivilgesellschaftliche Aufgaben relativ konstant bei 40 Prozent der BFD-Freiwilligen) besondere Aufmerksamkeit gilt. ${ }^{2}$ Untersucht werden sollen die Wirkungen eines Freiwilligendienstes auf die Freiwilligen selbst (Mikroebene), auf die Organisationen in ihren Einsatzbereichen (Mesoebene) sowie auf den gesellschaftlichen Zusammenhalt im Sinne z. B. der Nachhaltigkeit und Förderung der Engagementbereitschaft nach Beendigung des Dienstes (Makroebene).

Das FÖJ und das FSJ wurden 1995/1996 erstmalig untersucht (Arnold/Wüstendörfer 1996 und Rahrbach/Wüstendörfer/Arnold 1998), die letzte Evaluation von FSJ und FÖJ erfolgte von 2003 bis 2005 (Engels/Leucht/Machalowski 2008). Seitdem hat sich nicht nur die Anzahl der Teilnehmerinnen und Teilnehmer wesentlich erhöht, sondern auch die Gruppe der Freiwilligen und der zum Engagement bereiten Menschen sowohl soziodemographisch wie soziokulturell verändert. Die jetzige Evaluation knüpft an die früheren Ergebnisse an, arbeitet diese auf und entwickelt sie weiter.

Die Evaluation hat als umfassendes Forschungsprojekt vielfältige Bestandteile (u. a. Untersuchung der Strukturen der Freiwilligendienste, Expert/inneninterviewsund Gruppendiskussionen) und läuft insgesamt bis Ende 2015. Der Fokus wird auf die Freiwilligen in FSJ, FÖJ und BFD gelegt: In einer Längsschnittstudie werden sie dreimal über einen Zeitraum von drei Jahren unter anderem nach ihren Erwartungen und Motiven, aber auch nach den Erfahrungen, Bewertungen und Bildungs- und Lerneffekten des Dienstes gefragt. Die erste Befragung fand zu Beginn ihres Freiwilligendienstes bereits im Herbst 2012 statt, wobei die Freiwilligen unter 27 Jahren in 230 Einführungsseminaren aufgesucht wurden, während die Befragung der über 27-jährigen Freiwilligen im BFD postalisch erfolgte. Die zweite Befragung der Freiwilligen fand als Online-Befragung zum Ende des Freiwilligendienstes statt und wurde im Zeitraum von Juni bis September 2013 durchgeführt. Eine dritte Befragung der Freiwilligen wird im Herbst/Winter 2014, rund anderthalb Jahre nach Dienstende, stattfinden, um auch nachhaltige Wirkungen zu erfassen.

Auf einer Zwischentagung Mitte November 2013 wurden die Ergebnisse aus der ersten und zweiten Befragung der Freiwilligen vorgestellt und diskutiert (Huth/

2 Die Statistik dazu wird monatlich veröffentlicht unter http://www.bafza.de/presse/statistikeno.html 
Engels/Aram 2013). An der Erstbefragung zum Dienstbeginn nahmen 8.385 Befragte teil, davon 45 Prozent im FSJ, 11 Prozent im FÖJ und 30 Prozent im BFD unter 27 sowie 14 Prozent im BFD Ü 27. Von den Befragten waren 86 Prozent im sozialen Bereich (einschließlich Sport, Kultur und Denkmalpflege) und 14 Prozent im ökologischen Bereich engagiert. An der zweiten Befragung zum Dienstende nahmen noch knapp 3000 Freiwillige teil; die Verteilung auf die FreiwilligendienstFormate und auf die Einsatzbereiche war hier ähnlich.

Betrachtet man nun die Ergebnisse aus diesen ersten beiden Befragungen der Freiwilligen, so lassen sich folgende erste Thesen aufstellen: Die Freiwilligendienste werden insgesamt ausgewogener (Geschlecht, Alter, Schulabschluss). Durch die Altersöffnung im BFD werden neue Bevölkerungsschichten erreicht. 60 Prozent der befragten Freiwilligen sind weiblich, der BFD hat mit 50 Prozent männlichen Freiwilligen bei den unter 27-jährigen eine ausgeglichene Geschlechterverteilung. Im FSJ ist nach wie vor der Anteil der weiblichen Teilnehmenden am höchsten (Huth/Engels/Aram 2013: 16). Wenn man in Betracht zieht, dass bei der FSJ-Studie von 1998 sogar 90,7 Prozent der Teilnehmenden im FSJ weiblich waren (Rahrbach/Wüstendörfer/Arnold 1998), so ist auch hier eine Öffnung festzustellen. Insgesamt ist eine Zunahme des Anteils der männlichen Freiwilligen seit den 1990er Jahren zu verzeichnen.

Abb. 1: Geschlechterverteilung

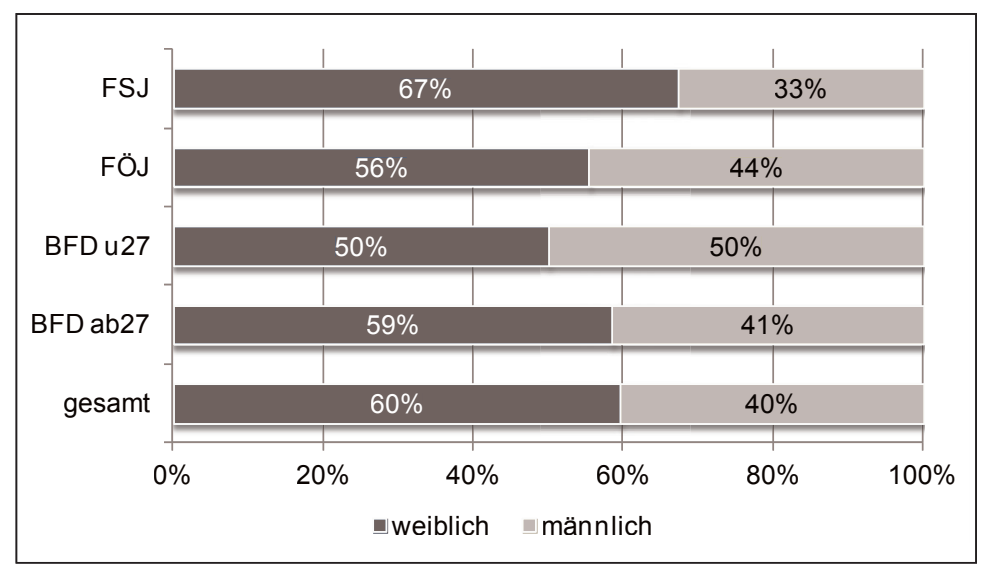

Quelle: Huth/Engels/Aram 2013:16

Auch was die Schulabschlüsse angeht, ist eine Veränderung festzustellen: 59 Prozent der Freiwilligen bei der aktuellen Evaluation gaben an, über (Fach-)Abitur 
zu verfügen. Bei der FSJ-Studie von 1998 hatten 61,5 Prozent Abitur (Rahrbach/ Wüstendörfer/Arnold 1998). Sieht man sich die Zahlen genauer an, so ist es vor allem die neue Gruppe der Ü 27 im BFD, die hier zu einer Öffnung der Freiwilligendienste für neue Zielgruppen führt: Während im FSJ, FÖJ und BFD unter 27 der Anteil der Freiwilligen, die über (Fach-)Abitur verfügen, weit über dem Bundesdurchschnitt liegt (allein im FÖJ mit 73 Prozent!), sind Freiwillige mit diesem höchsten Schulabschluss bei den über 27-jährigen Freiwilligen mit 22 Prozent unterrepräsentiert. Diese älteren Freiwilligen verfügen zu 43 Prozent über einen mittleren Schulabschluss und zu 29 Prozent über einen Hauptschulabschluss. Es wird deutlich, dass über die Öffnung des BFD für diese neue Altersgruppe andere gesellschaftliche Schichten für die Freiwilligendienste angesprochen werden.

Abb. 2: Schulabschluss der Freiwilligen

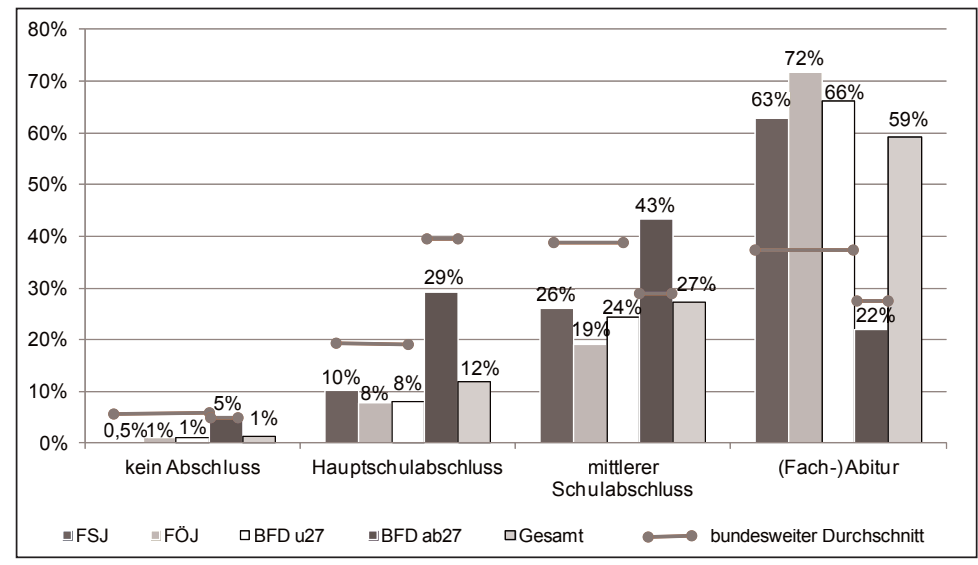

Quelle: Huth/Engels/Aram 2013:17

Sieht man sich an, welcher Tätigkeit die Freiwilligen vor Ihrem Dienst nachgegangen sind, so verhärtet sich die Annahme, dass bei den Ü 27 neue gesellschaftliche Gruppen angesprochen werden: Während die jungen Freiwilligen in FSJ, FÖJ und im BFD unter 27 Jahren zu 70 bis 80 Prozent angaben, vor dem Dienst die Schule beendet zu haben, waren von den über 27-jährigen Freiwilligen im BFD 51 Prozent im ALG-II-Bezug, 12 Prozent waren Rentner/innen, 11 Prozent waren vorher erwerbstätig und 5 Prozent waren vorher in Familienphase/Elternzeit (Huth/ Engels/Aram 2013:18).

Diese Zahlen machen eines deutlich: Wie mit dieser neuen Gruppe der über 27-jährigen Freiwilligen, ihren Hoffnungen und Erwartungen an einen Freiwilligendienst 
umgegangen werden soll, wird die große Herausforderung, aber auch Chance der kommenden Jahre sein.

Die sehr hohen Erwartungen der Freiwilligen werden im Einsatz nicht nur erreicht, sondern in fast allen Punkten sogar übertroffen.

Die Freiwilligen wurden in der ersten Befragung zu Beginn des Freiwilligendienstes gefragt, welche Erwartungen sie an ihr freiwilliges Engagement haben. In der zweiten Befragung zum Ende des Freiwilligendienstes wurde nachgehakt, inwieweit diese Erwartungen sich mit den gemachten Erfahrungen decken. Die Auswertung ergab, dass die Freiwilligen bereits mit sehr hohen Erwartungen in den Freiwilligendienst gehen, und dass diese hohen Erwartungen insbesondere bei den jüngeren Freiwilligen alle mehr als erwartet erfüllt wurden.

Zum Ende des Dienstes gaben beispielsweise 95 Prozent der unter 27-jährigen in FSJ, FÖJ und BFD an, Erfahrungen in einem neuen Bereich gesammelt zu haben, 92 Prozent hatten verantwortungsvolle Aufgaben übernommen und 92 Prozent gaben an, ihre persönlichen Fähigkeiten verbessert zu haben. Die Mehrheit hat während des Dienstes zahlreiche persönliche Erfahrungen gesammelt und konnte ihre persönlichen Fähigkeiten verbessern. 69 Prozent sammelten Anregungen zur Berufswahl bzw. sahen ihre Erwartungen, was Verbesserung der beruflichen Chancen angeht, erfüllt.

Abb. 3: Erwartungen und Erfahrungen der Freiwilligen unter 27 Jahren (Auswahl - Zustimmung: trifft voll zu und trifft eher zu)

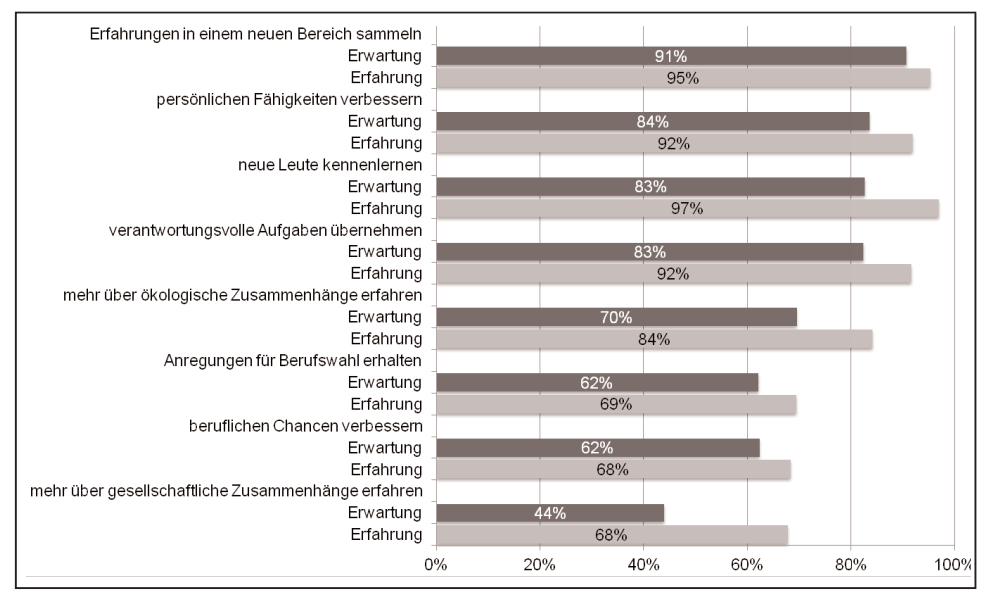


Bei den über 27-jährigen Freiwilligen im BFD gab es eine etwas andere Schwerpunktsetzung. Bei ihnen stand die Erwartung, gebraucht zu werden, im Vordergrund, und wurde mit 94 Prozent ebenfalls mehr als erwartet erfüllt. Auch sie gingen mit sehr hohen Erwartungen in den Dienst, und auch bei Ihnen wurden fast alle Erwartungen übertroffen. Eine wichtige Ausnahme fällt dabei ins Auge: Während 41 Prozent der Befragten zu Beginn ihres Dienstes erklärten, sie erwarteten, ihre beruflichen Chancen durch den Freiwilligendienst zu verbessern, gaben nur 31 Prozent zum Ende des Dienstes an, dass diese Erfahrung auch gemacht wurde. Auch hier zeigt sich wieder die Besonderheit dieser neuen Gruppe von Freiwilligen.

Abb. 4: Erwartungen und Erfahrungen der Freiwilligen ab 27 Jahren (Auswahl - Zustimmung: trifft voll zu und trifft eher zu)

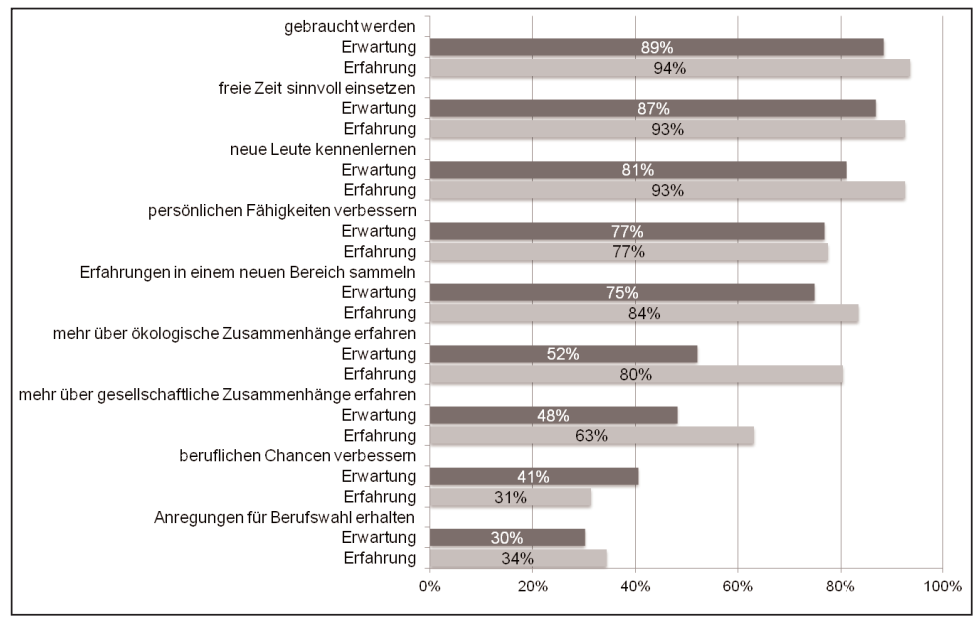

Quelle: Huth/Engels/Aram 2013: 21

Zwischen BFD, FSJ und FÖJ sind keine Qualitätsunterschiede festzustellen. Alle drei Dienste werden gleichermaßen gut bewertet.

Ein sehr interessantes und erfreuliches Ergebnis dieser ersten Auswertungen war, dass es bei den Freiwilligen in FSJ, FÖJ und BFD wenig Unterschiede in der Beurteilung ihres Freiwilligendienstes gab. Insgesamt waren 85 Prozent der befragten Freiwilligen mit ihrer Tätigkeit im FSJ, FÖJ bzw. BFD sehr bzw. eher zufrieden. Die Freiwilligen im FSJ und im BFD über 27 gaben mit 55 Prozent am häufigsten an, dass sie mit ihrer Tätigkeit sehr zufrieden sind. Im BFD unter 27 ist der Anteil mit 47 Prozent etwas geringer, im FÖJ mit 44 Prozent am geringsten. 
Abb. 5: Zufriedenheit mit der Tätigkeit

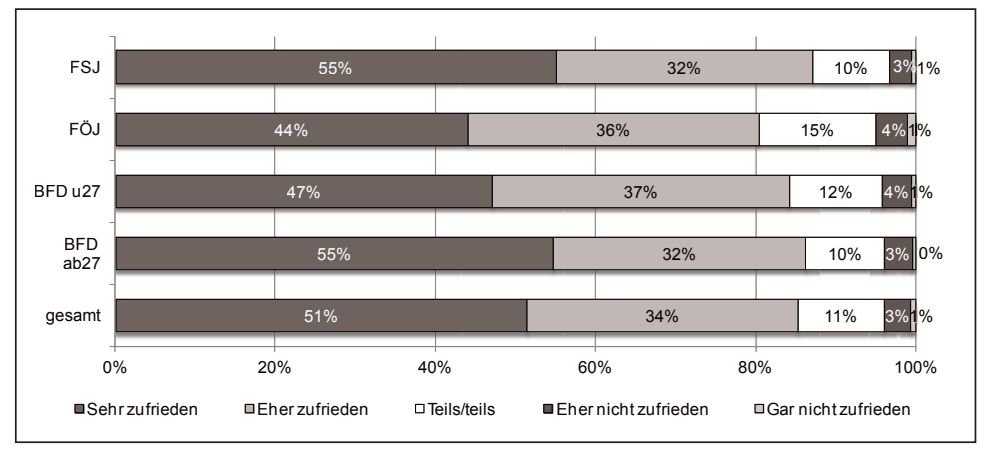

Quelle: Huth/Engels/Aram 2013: 21

Bezüglich der Bewertung der Seminare erklärten 90 Prozent der Befragten im FÖJ, diese sehr gut oder gut zu finden, 80 Prozent im BFD ab 27, 76 Prozent im FSJ und 72 Prozent im BFD unter 27 teilten diese Einschätzung.

88 Prozent der befragten Freiwilligen würden einen Freiwilligendienst weiterempfehlen, darunter 91 Prozent der Befragten im FÖJ, 89 Prozent im FSJ und 86 Prozent BFD unter 27 bzw. 83 Prozent im BFD über 27 (Huth/Engels/Aram 2013: 23).

\section{Fazit}

Die ersten Ergebnisse machen deutlich, dass die Öffnung der Freiwilligendienste für neue Altersgruppen zu einer Ausweitung der Teilnehmendenschaft hinsichtlich sozialer Herkunft und Bildung geführt hat. Zudem ist eine Angleichung der Geschlechterverteilung festzustellen; dies betrifft vor allem den BFD unter 27 Jahren, gilt aber auch für FÖJ und FSJ. Für alle drei Formate gilt, dass die Freiwilligen sich mit sehr hohen Erwartungen an ihren Freiwilligendienst engagieren, und diese Erwartungen werden zum allergrößten Teil sogar übertroffen. Wenn man sich vor Augen hält, dass in den letzten Jahren eine Vervielfachung der Freiwilligenzahlen auf derzeit knapp 100.000 Freiwillige in FSJ, FÖJ und BFD stattgefunden hat, und das bei gleichbleibendem Zufriedenheitsniveau, ist das schon eine beachtliche Aussage.

Insgesamt geben die ersten Befragungsergebnisse viele Anregungen, werfen aber auch viele Fragen für die weitere Auswertungen der Befragungen und qualitativen Nachuntersuchungen auf. Im weiteren Verlauf der Evaluation wird es unter anderem darum gehen, die Angaben zu den Rahmenbedingungen des 
Freiwilligendienstes, wie z. B. das Taschengeld, auszuwerten. Weiterhin werden Auswertungen zu ausgewählten Zielgruppen durchgeführt. So soll beispielsweise geprüft werden, ob sich die Angaben zwischen Teilnehmenden mit und ohne Migrationshintergrund unterscheiden. Auch weitere Vergleiche zwischen den Angaben in der ersten Befragung zu Beginn des Freiwilligendienstes und der zweiten Befragung zum Dienstende werden vorgenommen, um Veränderungen während der Zeit des Freiwilligendienstes abbilden zu können. Außerdem ist geplant, die Angaben auf individueller Ebene zu verknüpfen, um Längsschnittauswertungen durchführen zu können. Der Abschlussbericht Ende 2015 wird daher noch viele weitere, wichtige Erkenntnisse bieten.

\section{Literatur}

Arnold, Thomas und Werner Wüstendörfer (1996): Abschlußbericht zum Freiwilligen Ökologischen Jahr, Stuttgart.

BAFzA/Bundesamt für Familie und zivilgesellschaftliche Aufgaben (2013), http://www. bafza.de/fileadmin/redaktion/downloads/Abt2/201/BL-Alter_u_Geschlecht.pdf (Zugriff am 30. Dezember 2013). http://www.bundesfreiwilligendienst.de/fileadmin/ de.bundesfreiwilligendienst/content.de/Service_Menue_Kopf/Presse/Statistiken/BFD_ nach_Alter_und_Geschlecht.pdf

Engels, Dietrich, Martina Leucht und Gerhard Machalowski (2008): Evaluation des freiwilligen sozialen Jahres. Empirische Studien zum bürgerschaftlichen Engagement, hrsg. vom Bundesministerium für Familie, Senioren, Frauen und Jugend, Wiesbaden.

Huth, Susanne (2013): Hintergrund, Ziele und Methoden der gemeinsamen Evaluation des Gesetzes über den Bundesfreiwilligendienst (BFDG) und des Gesetzes zur Förderung von Jugendfreiwilligendiensten (JFDG), in: Voluntaris 1/2013, S. 139-143.

Huth, Susanne, Dietrich Engels und Elisabeth Aram (2013): Präsentation erster Ergebnisse der Evaluation von Bundesfreiwilligendienst und Jugendfreiwilligendiensten, in: BMFSFJ: Fachtagung „Freiwillig gestalten - erste Evaluationsergebnisse und aktuelle Entwicklungen der Freiwilligendienste" Tagungsdokumentation, 18. und 19. November 2013, S. 12-27, Berlin

Rahrbach, Andrea, Werner Wüstendörfer und Thomas Arnold (1998): Untersuchung zum Freiwilligen Sozialen Jahr, Stuttgart. 